\title{
Inverse Spectral Problems for Second-Order Difference Operators and Their Application to the Study of Volterra Type Systems
}

\begin{abstract}
A. S. Osipov
In this paper, some links between inverse problem methods for the second-order difference operators and nonlinear dynamical systems are studied. In particular, the systems of Volterra type are considered. It is shown that the classical inverse problem method for semi-infinite Jacobi matrices can be applied to obtain a hierarchy of Volterra lattices, and this approach is compared with the one based on Magri's bi-Hamiltonian formalism. Then, using the inverse problem method for nonsymmetric difference operators (which amounts to reconstruction of the operator from the moments of its Weyl function), the hierarchies of Volterra and Toda lattices are studied. It is found that the equations of Volterra hierarchy can be transformed into their Toda counterparts, and this transformation can be easily described in terms of the above-mentioned moments.
\end{abstract}

Keywords: inverse spectral problems, difference operators, Jacobi matrices, Volterra lattices, Toda lattices

\section{Introduction}

Since the classical works of Kac, van Moerbeke and Moser [1, 2] the inverse spectral problems for difference operators have been applied for integration of nonlinear dynamical systems. A typical example of such application is the work of Berezanski [7], where the semi-infinite Toda lattice was integrated by means of the classical inverse spectral problem for Jacobi operators. This work inspired the development of the inverse spectral problem methods with an aim to cover wider classes of nonlinear systems. One of the advantages of these methods is that they impose loose restrictions on the operator's coefficients and the inverse problem data. Also, since the inverse spectral problems for difference operators are connected with the theory of continued fractions, Hermite-Pade approximations and orthogonal polynomials, the development of integration methods for nonlinear systems had an influence on these "neighboring" areas, see [12? ].

Received December 12, 2019

Accepted July 23, 2020

Andrey S. Osipov

osipa68@yahoo.com

Scientific Research Institute for System Analysis of the Russian Academy of Sciences

Nakhimovskii pr. 36-1, Moscow, 117218 Russia

RUSSIAN JOURNAL OF NONLINEAR DYNAMICS, 2020, 16(3), 397-419 
Our aim here is to look at some issues arising in the study of integrable systems from the inverse spectral theory point of view. Although most of these issues are not new, the proposed approach allows generalization (namely, to the case of systems with matrix (or operator) coefficients).

In this paper, we mainly consider the Volterra lattice (also referred to, due to its physical applications, as Langmuir lattice), which is a typical object of study in the theory of integrable systems. This system has a number of interesting properties, for example, it can be considered as a discrete analog of the Korteweg-de Vries equation, see [9, 12, 16]. It also appears in the discretization of conformal field theory; the Poisson bracket for this system, found by Faddeev and Takhtajan, provides a lattice generalization of a certain Virasoro algebra [5, 20].

The paper is organized as follows. The next section contains a brief summary of the inverse spectral problem method for the Jacobi operators (we skipped some details, e.g., those related to $J$-continued fractions, which are not relevant to our further discussion). In Section 3 it is shown how, using this method, one can construct a hierarchy of Volterra lattices, and the proposed approach is compared with the construction method based on Magri's bi-Hamiltonian formalism [15, 16]. In Section 4, using an inverse spectral method for nonsymmetric difference operators of the second order, we build up the hierarchies of Volterra and Toda systems and show how the systems of Volterra hierarchy can be transformed into Toda-type systems. Here we mention that a similar transformation between the finite Volterra and Toda lattices was established in [10], which allows one to transform the Poisson brackets, master symmetries,first integrals of a given Volterra lattice to the similar objects of the corresponding Toda lattice. We describe this transformation in terms of the inverse spectral data of Lax operators, corresponding to both types of systems. Also we outline the application of our approach to the finite systems of Volterra and Toda type.

\section{Inverse problems for Jacobi operators}

First we recall some basic facts and definitions from the theory of Jacobi operators, essential for further consideration [3, 7]. Namely, consider the semi-infinite Jacobi matrix

$$
J=\left(\begin{array}{cccc}
b_{0} & a_{0} & 0 & 0 \\
a_{0} & b_{1} & a_{1} & 0 \\
0 & \ddots & \ddots & \ddots
\end{array}\right)
$$

such that

$$
a_{i}>0, \quad b_{i} \in \mathbb{R}, \quad i \in \mathbb{Z}_{+} .
$$

Denote by $l^{2}$ the Hilbert space of complex quadratic summable sequences and by $\left(e_{n}\right)_{n=0}^{\infty}$ its standard orthonormal basis. The matrix $J$ generates the (minimal) closed symmetric operator in $l^{2}$ and in the basis $\left(e_{n}\right)$ this operator (also denoted by $J$ ) has the matrix representation $J$. For the operator $J$, its spectral function is defined as: $\rho(\lambda)=\left(E_{\lambda} e_{0}, e_{0}\right)$, where $E_{\lambda}$ is the spectral measure of a certain self-adjoint extension $J_{\text {ext }}$ of $J$ (i. e., according to the spectral theorem for self-adjoint operators, $J \subset J_{\text {ext }}=\int_{-\infty}^{\infty} \lambda d E_{\lambda}$; more details and definitions can be found in $\left.[3,6]\right)$. The Weyl function of $J$ is defined as the Stieltjes transform of $\rho(\lambda): m(z)=\int_{-\infty}^{\infty} \frac{d \rho(\lambda)}{\lambda-z}$. In terms of the inverse spectral problems, $m(z)$ is the object equivalent to $\rho(\lambda)$. 
The inverse spectral problem for the operator $J$ can be formulated as follows: given $\rho(\lambda)$ or $m(z)$, find $a_{i}$ and $b_{i}$ (restore the matrix $J$ ).

For the moments $s_{n}:=\int_{-\infty}^{\infty} \lambda^{n} d \rho(\lambda), n \in \mathbb{Z}_{+}$we have

$$
s_{n}=\int_{-\infty}^{\infty} \lambda^{n} d\left(E_{\lambda} e_{0}, e_{0}\right) \stackrel{\text { by the spectral theorem }}{=}\left(J^{n} e_{0}, e_{0}\right)=\left(J^{n}\right)_{0,0}
$$

(the latter notation is for the matrix $J$ ). In fact, these moments are related to the asymptotic expansion of the resolvent $R_{z}:=(z E-J)^{-1}$ of the operator $J$ at infinity, because for sufficiently large $z$ we have $(z E-J)^{-1} \sim \frac{E}{z}+\frac{J}{z^{2}}+\frac{J^{2}}{z^{3}}+\ldots$ By the spectral theorem, $m(z)=\left(R_{z} e_{0}, e_{0}\right)$. Thus, $m(z)$ is a resolvent function of the operator $J$, and we can call the sequence $S=\left\{s_{j}\right\}_{j=0}^{\infty}$ the moment sequence corresponding to the Weyl function $m(z)$. Also note that although there may be different self-adjoint operator extensions corresponding to the same matrix $J$ (and therefore different $\rho(\lambda)$ and $m(z)$ ), the sequence $S$ is unique for the matrix $J$.

As follows from the Hamburger theorem (its detailed proof is contained, e. g., in [3]), $S$ is a positive sequence, which means that for all $n$

$$
\Delta_{n}(J)>0
$$

where $\Delta_{n}(J)$ are determinants of the Hankel matrices $H_{n}=\left(s_{i+j}\right)_{i, j=0}^{n}$. The positiveness of $S$ implies that a corresponding $\rho(\lambda)$ defined on $\mathbb{R}$ has infinitely many growth points. Also, $\rho(\lambda)$ is a nondecreasing function (this fact follows from its definition and the spectral theorem).

To the matrix $J$ we assign the second-order difference equation:

$$
a_{n-1} y_{n-1}+b_{n} y_{n}+a_{n} y_{n+1}=\lambda y_{n}, \quad \lambda \in \mathbb{C}, \quad n=1,2, \ldots
$$

It has two linearly independent systems of solutions:

$$
\begin{gathered}
P(\lambda):=\left(P_{n}(\lambda)\right)_{n=0}^{\infty} \quad \text { and } \quad Q(\lambda):=\left(Q_{n}(\lambda)\right)_{n=0}^{\infty} ; \\
P_{0}(\lambda)=1, \quad P_{1}(\lambda)=\frac{\lambda-b_{0}}{a_{0}} ; \quad Q_{0}(\lambda)=0, \quad Q_{1}(\lambda)=\frac{1}{a_{0}} .
\end{gathered}
$$

Thus, $P_{n}(\lambda)$ and $Q_{n}(\lambda)$ are polynomials in $\lambda$, and any solution $\left(y_{n}(\lambda)\right)_{n=0}^{\infty}$ is a linear combination of them: $y_{n}=P_{n}(\lambda) c+Q_{n}(\lambda) d, c, d \in \mathbb{C}$. We write $P_{n}(\lambda)=p_{0, n}+p_{1, n} \lambda+\ldots+p_{n, n} \lambda^{n} ; p_{n, n} \neq 0$. The polynomials $P_{n}(\lambda)$ are orthogonal with respect to $S$ (or $d \rho(\lambda)$ ):

$$
\sum_{i, j=0}^{n+m} p_{i, n} p_{j, m} s_{i+j}=\int_{-\infty}^{\infty} P_{n}(\lambda) P_{m}(\lambda) d \rho(\lambda)=\delta_{m, n} .
$$

This can be proved in the following manner. Using Eq. (2.2), for the vectors $e_{n}$ we get $e_{n}=$ $=P_{n}(J) e_{0}$. Then we have

$$
\begin{aligned}
& \delta_{m, n}=\left(e_{m}, e_{n}\right)=\left(P_{m}(J) e_{0}, P_{n}(J) e_{0}\right)=\left(P_{n}(J) P_{m}(J) e_{0}, e_{0}\right)= \\
& \text { by the spectral theorem } \int_{-\infty}^{\infty} P_{n}(\lambda) P_{m}(\lambda) d\left(E_{\lambda} e_{0}, e_{0}\right)=\int_{-\infty}^{\infty} P_{n}(\lambda) P_{m}(\lambda) d \rho(\lambda) .
\end{aligned}
$$


Using the above relations, we get a solution of the inverse problem: given $\rho(\lambda)$ and the sequence of powers $1, \lambda, \lambda^{2}, \ldots$ we construct the polynomials $P_{n}(\lambda)$ with leading positive coefficients by applying the standard orthogonalization procedure. From this orthogonality and Eq. (2.2) it follows that

$$
a_{n}=\int_{-\infty}^{\infty} \lambda P_{n}(\lambda) P_{n+1}(\lambda) d \rho(\lambda), \quad b_{n}=\int_{-\infty}^{\infty} \lambda P_{n}^{2}(\lambda) d \rho(\lambda) .
$$

Also, the orthogonality relations lead to the following representation of $P_{n}(\lambda)$ :

$$
P_{n}(\lambda)=\sqrt{\frac{1}{\Delta_{n-1}(J) \Delta_{n}(J)}}\left|\begin{array}{cccc}
s_{0} & s_{1} & \ldots & s_{n} \\
s_{1} & s_{2} & \ldots & s_{n+1} \\
\vdots & \vdots & \ldots & \vdots \\
s_{n-1} & s_{n} & \ldots & s_{2 n-1} \\
1 & \lambda & \ldots & \lambda^{n}
\end{array}\right| .
$$

Hence, $P_{n}(\lambda)=\sqrt{\frac{\Delta_{n-1}(J)}{\Delta_{n}(J)}} \lambda^{n}+R_{n-1}(\lambda)$ and it follows from Eq. (2.2) that

$$
a_{n}=\frac{\sqrt{\Delta_{n+1}(J) \Delta_{n-1}(J)}}{\Delta_{n}(J)}, \quad b_{n}=\frac{D_{n}(J)}{\Delta_{n}(J)}-\frac{D_{n-1}(J)}{\Delta_{n-1}(J)},
$$

where

$$
D_{n}(J)=\operatorname{det}\left(\begin{array}{cccc}
s_{0} & \ldots & s_{n-1} & s_{n+1} \\
\vdots & & \vdots & \vdots \\
s_{n} & \ldots & s_{2 n-1} & s_{2 n+1}
\end{array}\right), \quad \Delta_{-1}=1, \quad D_{-1}=0, \quad D_{0}(J)=s_{1} .
$$

Thus, Eqs. (2.6) give the reconstruction procedure of the elements of $J$ in terms of the moment sequence $S$, and this procedure amounts to the solution of the inverse spectral problem. In other words, we are coming to the following formalization of the latter:

$$
S=\left\{\left(J^{k}\right)_{0,0}\right\}_{k=0}^{\infty} \rightarrow J .
$$

Condition (2.1), together with $s_{0}=0$, gives a criterion for an arbitrary sequence $S=\left\{s_{k}\right\}_{k=0}^{\infty}$ of integers to be the moment sequence of a certain Jacobi matrix $J$.

Using the second of equations (2.6) and applying induction on $n$, we obtain the following criterion of "sparsity" of the matrix $J$ in terms of $S$ :

Proposition 1. $\left\{b_{n}=0\right\}_{n=0}^{\infty} \Longleftrightarrow\left\{s_{2 k+1}=0\right\}_{k=0}^{\infty}$.

Therefore, for the matrix $J$ with zero main diagonal, all odd moments of $d \rho(\lambda)$ (or the Weyl function of $J$ ) are zero. Also, as noted by Moser in [2], for such $J$ the corresponding Weyl function $m(z)$ is odd.

Most of the above results have analogs for nonsymmetric difference operators (e.g., operators generated by infinite three diagonal matrices, see Section 4), including the operators with matrix coefficients. 


\section{Integration of semi-infinite Volterra lattices}

Consider the Cauchy problem for the system:

$$
\begin{gathered}
\dot{b}_{i}=b_{i}\left(b_{i+1}-b_{i-1}\right), \quad i \in \mathbb{Z}_{+}, \quad b_{i}=b_{i}(t), \quad t \in[0, T), \quad 0<T \leqslant \infty ; \\
b_{i}(t) \in l_{\infty}, \quad b_{-1}=0 .
\end{gathered}
$$

Assume that for all $t, b_{i}(t)>0$ and set $b_{i}=a_{i}^{2}, a_{i}>0$. Then the system can be rewritten as

$$
\dot{a}_{i}=\frac{1}{2} a_{i}\left(a_{i+1}^{2}-a_{i-1}^{2}\right) .
$$

This system admits the Lax representation $\dot{L}=[L, A]$ with the following infinite matrices $L=L(t)$ and $A=A(t)[1]:$

$$
\begin{aligned}
& L(t)=\left(\begin{array}{cccccc}
0 & a_{0} & 0 & 0 & 0 & \\
a_{0} & 0 & a_{1} & 0 & 0 & \\
0 & a_{1} & 0 & a_{2} & 0 & \\
0 & \ddots & \ddots & \ddots & \ddots & \ddots
\end{array}\right), \\
& A(t)=\frac{1}{2}\left(\begin{array}{ccccc}
0 & 0 & -a_{0} a_{1} & 0 & 0 \\
0 & 0 & 0 & -a_{1} a_{2} & 0 \\
a_{0} a_{1} & 0 & 0 & 0 & -a_{2} a_{3} \\
0 & a_{1} a_{2} & \ddots & \ddots & \ddots
\end{array}\right) .
\end{aligned}
$$

Thus, the matrix $L(t)$ is a Jacoby matrix with zero main diagonal, and all previous results are valid here. Note that $A(t)$ is a skew-symmetric part of $L^{2}(t) / 2$, i.e.,

$$
A(t)=\operatorname{skew}\left(L^{2}(t) / 2\right)=\frac{1}{2}\left(L_{\text {low }}^{2}(t)-L_{u p}^{2}(t)\right),
$$

where $L_{\text {low }}^{2}(t)$ and $L_{\text {up }}^{2}(t)$ are, respectively, strict lower and upper triangular parts of $L^{2}(t)$. Using the above Lax representation and the evolution of polynomials $P(\lambda, t)$ and $Q(\lambda, t)$ corresponding to $L(t)$, one may get the following equation for its Weyl function, see e.g. [6]:

$$
\dot{m}(z, t)=m(z, t)\left(z^{2}-a_{0}^{2}(t)\right)+z=m(z, t)\left(z^{2}-s_{2}(t)\right)+z .
$$

Since $m(z, t)$ is an odd function, we have: $m(z, t)=0.5(m(z, t)-m(-z, t))=z \int_{-\infty}^{\infty} \frac{d \rho(\lambda, t)}{\lambda^{2}-z^{2}}$. We therefore find:

$$
\begin{aligned}
\dot{m}(z, t) & =\left(z \int_{-\infty}^{\infty} \frac{d \rho(\lambda, t)}{\lambda^{2}-z^{2}}\right)=\left(z \int_{-\infty}^{\infty} \frac{\left(z^{2}-a_{0}^{2}(t)\right) d \rho(\lambda, t)}{\lambda^{2}-z^{2}}\right)+z \int_{-\infty}^{\infty} d \rho(\lambda, t)= \\
& =z \int_{-\infty}^{\infty} \frac{\left(\lambda^{2}-a_{0}^{2}(t)\right) d \rho(\lambda, t)}{\lambda^{2}-z^{2}} .
\end{aligned}
$$


Using the uniqueness of the Stieltjes transform, we get the following evolutionary equation for the spectral function of $L(t)$ :

$$
\dot{d} \rho(\lambda, t)=\left(\lambda^{2}-a_{0}^{2}(t)\right) d \rho(\lambda, t)=\left(\lambda^{2}-s_{2}(t)\right) d \rho(\lambda, t) .
$$

Therefore,

$$
d \rho(\lambda, t)=e^{\lambda^{2} t} d \rho(\lambda, 0) e^{-\int_{0}^{t} a_{0}^{2}(\tau) d \tau} .
$$

Using the normalization condition for $d \rho(\lambda, t)$, we find

$$
1=\int_{-\infty}^{\infty} d \rho(\lambda, t)=e^{-\int_{0}^{t} a_{0}^{2}(\tau) d \tau} \int_{-\infty}^{\infty} e^{\lambda^{2} t} d \rho(\lambda, 0)
$$

and, finally,

$$
d \rho(\lambda, t)=\frac{e^{\lambda^{2} t} d \rho(\lambda, 0)}{\int_{-\infty}^{\infty} e^{\lambda^{2} t} d \rho(\lambda, 0)}
$$

Equation (3.6) leads to the corresponding equation for the moments:

$$
\dot{s}_{2 k}=s_{2(k+1)}-s_{2} s_{2 k}, \quad k \in \mathbb{Z}_{+} .
$$

Note that this formula is similar (up to a constant) to the formula (2.2) from the classical paper of M. Kac and P. van Moerbeke [1].

In view of the above, we can apply the procedure of recovering the elements $a_{i}(t)$ from $d \rho(\lambda, t)$, thus solving the Volterra system.

Theorem 1. Suppose we have a nondecreasing function $\rho(\lambda, t)$ defined on the Borel sets of $\mathbb{R}$, with infinitely many growth points and such that all its moments $s_{k}(t)=\int_{-\infty}^{\infty} \lambda^{k} d \rho(\lambda, t)$ are finite, $s_{2 k+1}(t)=0, k \in \mathbb{Z}_{+} ; s_{0}(t)=\int_{-\infty}^{\infty} d \rho(\lambda, t)=1$. Also suppose that $d \rho(\lambda, t)$ satisfies Eq. (3.6) for all $t \in[0, T)$. Then

$$
a_{i}(t)=\int_{-\infty}^{\infty} \lambda P_{i}(\lambda, t) P_{i+1}(\lambda, t) d \rho(\lambda, t),
$$

where $\left\{P_{i}(\lambda, t)\right\}_{i=0}^{\infty}$ are the polynomials orthogonal with respect to $d \rho(\lambda, t)$ and satisfy the system (3.2).

Proof. Since $\left\{P_{i}(\lambda, t)\right\}_{i=0}^{\infty}$ is an orthonormal system with respect to $d \rho(\lambda, t)$, we get

$$
\lambda P_{i}(\lambda, t)=a_{i-1}(t) P_{i-1}(\lambda, t)+a_{i}(t) P_{i+1}(\lambda, t), \quad i=1,2, \ldots
$$

(one may consider $a_{i}(t)$ as the Fourier coefficients of polynomials $\lambda P_{i}(\lambda, t)$ with respect to this system. Since $s_{2 k+1}=s_{2 k+1}(t)=0$, using Eq. (2.5) one can check that the polynomials $P_{i}(\lambda, t)$ contain only even powers of $\lambda$, thus $\left.\int_{-\infty}^{\infty} \lambda P_{i}(\lambda, t) P_{i}(\lambda, t) d \rho(\lambda, t)=0\right)$. 
Then, differentiating Eq. (3.7), we have

$$
\begin{aligned}
\dot{a}_{i}(t) & =\left(\int_{-\infty}^{\infty} \lambda P_{i}(\lambda, t) P_{i+1}(\lambda, t) d \rho(\lambda, t)\right)=\int_{-\infty}^{\infty} \lambda P_{i}(\lambda, t) P_{i+1}(\lambda, t) d \dot{\rho}(\lambda, t)+ \\
& +\int_{-\infty}^{\infty} \lambda \dot{P}_{i}(\lambda, t) P_{i+1}(\lambda, t) d \rho(\lambda, t)+\int_{-\infty}^{\infty} \lambda P_{i}(\lambda, t) \dot{P}_{i+1}(\lambda, t) d \rho(\lambda, t)=\mathrm{I}+\mathrm{II}+\mathrm{III} .
\end{aligned}
$$

Using Eq. (3.6), we find

$$
\begin{aligned}
I & =\int_{-\infty}^{\infty} \lambda^{3} P_{i}(\lambda, t) P_{i+1}(\lambda, t) d \rho(\lambda, t)-s_{2}(t) \int_{-\infty}^{\infty} \lambda P_{i}(\lambda, t) P_{i+1}(\lambda, t) d \rho(\lambda, t)= \\
& =\int_{-\infty}^{\infty} \lambda^{3} P_{i}(\lambda, t) P_{i+1}(\lambda, t) d \rho(\lambda, t)-s_{2}(t) a_{i}(t) .
\end{aligned}
$$

From Eq. (3.8) and orthogonality of $\left\{P_{i}(\lambda, t)\right\}$ it follows that

$$
\begin{aligned}
\mathrm{II} & =a_{i+1}(t) \int_{-\infty}^{\infty} \dot{P}_{i}(\lambda, t) P_{i+2}(\lambda, t) d \rho(\lambda, t)+a_{i}(t) \int_{-\infty}^{\infty} \dot{P}_{i}(\lambda, t) P_{i}(\lambda, t) d \rho(\lambda, t)= \\
& =a_{i+1}(t) \int_{-\infty}^{\infty} \sum_{k=0}^{i} f_{k}(t) P_{k}(\lambda, t) P_{i+2}(\lambda, t) d \rho(\lambda, t)+a_{i}(t) \int_{-\infty}^{\infty} \dot{P}_{i}(\lambda, t) P_{i}(\lambda, t) d \rho(\lambda, t)= \\
& =a_{i}(t) \int_{-\infty}^{\infty} \dot{P}_{i}(\lambda, t) P_{i}(\lambda, t) d \rho(\lambda, t),
\end{aligned}
$$

where $f_{k}(t)=\int_{-\infty}^{\infty} \dot{P}_{i}(\tau, t) P_{k}(\tau, t) d \rho(\lambda, t)$ are the Fourier coefficients of polynomials $\dot{P}_{i}(\lambda, t)$ with respect to the system $\left\{P_{i}(\lambda, t)\right\}$.

Differentiating $\int_{-\infty}^{\infty} P_{i}(\lambda, t) P_{i}(\lambda, t) d \rho(\lambda, t)=1$ by $t$, we get

$$
0=2 \int_{-\infty}^{\infty} \dot{P}_{i}(\lambda, t) P_{i}(\lambda, t) d \rho(\lambda, t)+\int_{-\infty}^{\infty} \lambda^{2} P_{i}(\lambda, t) P_{i}(\lambda, t) d \rho(\lambda, t)-s_{2}(t) .
$$

Thus, for all $i$

$$
\int_{-\infty}^{\infty} \dot{P}_{i}(\lambda, t) P_{i}(\lambda, t) d \rho(\lambda, t)=-\frac{1}{2}\left(\int_{-\infty}^{\infty} \lambda^{2} P_{i}^{2}(\lambda, t) d \rho(\lambda, t)-s_{2}(t)\right)
$$

and for II we finally find

$$
\mathrm{II}=\frac{a_{i}(t)}{2}\left(-\int_{-\infty}^{\infty} \lambda^{2} P_{i}^{2}(\lambda, t) d \rho(\lambda, t)+s_{2}(t)\right)
$$


For III we get from Eq. (3.8)

$$
\mathrm{III}=a_{i}(t) \int_{-\infty}^{\infty} \dot{P}_{i+1}(\lambda, t) P_{i+1}(\lambda, t) d \rho(\lambda, t)+a_{i-1}(t) \int_{-\infty}^{\infty} \dot{P}_{i+1}(\lambda, t) P_{i-1}(\lambda, t) d \rho(\lambda, t) .
$$

Differentiating by $t$ the relation $\int_{-\infty}^{\infty} P_{i+1}(\lambda, t) P_{i-1}(\lambda, t) d \rho(\lambda, t)=0$ and using Eq. (3.6), we find that

$$
\int_{-\infty}^{\infty} \dot{P}_{i+1}(\lambda, t) P_{i-1}(\lambda, t) d \rho(\lambda, t)=-\int_{-\infty}^{\infty} \lambda^{2} P_{i-1}(\lambda, t) P_{i+1}(\lambda, t) d \rho(\lambda, t) .
$$

Applying Eq. (3.9) to $\int_{-\infty}^{\infty} \dot{P}_{i+1}(\lambda, t) P_{i+1}(\lambda, t) d \rho(\lambda, t)$, we finally find that III can be written as

$$
\mathrm{III}=-\frac{a_{i}(t)}{2}\left(\int_{-\infty}^{\infty} \lambda^{2} P_{i+1}^{2}(\lambda, t) d \rho(\lambda, t)-s_{2}(t)\right)-a_{i-1}(t) \int_{-\infty}^{\infty} \lambda^{2} P_{i-1}(\lambda, t) P_{i+1}(\lambda, t) d \rho(\lambda, t) .
$$

Summing up I, II and III, we arrive at the following relation for $\dot{a}_{i}(t)$ :

$$
\begin{aligned}
\dot{a}_{i}(t) & =\int_{-\infty}^{\infty} \lambda^{3} P_{i}(\lambda, t) P_{i+1}(\lambda, t) d \rho(\lambda, t)- \\
& -\frac{a_{i}(t)}{2}\left(\int_{-\infty}^{\infty} \lambda^{2} P_{i+1}^{2}(\lambda, t) d \rho(\lambda, t)+\int_{-\infty}^{\infty} \lambda^{2} P_{i}^{2}(\lambda, t) d \rho(\lambda, t)\right)- \\
& -a_{i-1}(t) \int_{-\infty}^{\infty} \lambda^{2} P_{i-1}(\lambda, t) P_{i+1}(\lambda, t) d \rho(\lambda, t) .
\end{aligned}
$$

Again, applying Eq. (3.8) to get rid of $\lambda^{2}$ and $\lambda^{3}$ in Eq. (3.10), we finally obtain

$$
\dot{a}_{i}=a_{i-1}^{2} a_{i}+a_{i}^{3}+a_{i+1}^{2} a_{i}-\frac{a_{i}}{2}\left(a_{i-1}^{2}+2 a_{i}^{2}+a_{i+1}^{2}\right)-a_{i-1}^{2} a_{i}=\frac{1}{2} a_{i}\left(a_{i+1}^{2}-a_{i-1}^{2}\right) .
$$

Note that in the works of Bogoyavlenskii the first equations of Volterra hierarchy were derived from their common Lax representation with spectral parameter. Also, the links between the Volterra hierarchy and the hierarchy of Korteveg-de Vries (KdV) equations were studied there (see [9] for details). These links were considered in detail in [16], where it was finally established that the equations of the former hierarchy (named in [16] the KM hierarchy after M. Kac and P. van Moerbeke) represent a discretization of the equations of the latter one. Here the shape of Eq. (3.6) and the method of proving the above proposition lead us to another way of constructing this hierarchy. Namely, the following statement holds.

Theorem 2. There exists a countable set of nonlinear dynamical systems having the same matrix $L$ in their Lax representation as the system (3.2). The corresponding spectral functions satisfy the evolutionary equations:

$$
\dot{d} \rho(\lambda, t)=\left(\lambda^{2 k}-s_{2 k}(t)\right) d \rho(\lambda, t), \quad k \in \mathbb{N} .
$$


It can be proved in the following manner. Assuming that Eq. (3.11) holds true and applying the same arguments as those used in deriving Eq. (3.10), we get the following relation:

$$
\begin{aligned}
\dot{a}_{i}(t) & =\int_{-\infty}^{\infty} \lambda^{2 k+1} P_{i}(\lambda, t) P_{i+1}(\lambda, t) d \rho(\lambda, t)- \\
& -\frac{a_{i}(t)}{2}\left(\int_{-\infty}^{\infty} \lambda^{2 k} P_{i+1}^{2}(\lambda, t) d \rho(\lambda, t)+\int_{-\infty}^{\infty} \lambda^{2 k} P_{i}^{2}(\lambda, t) d \rho(\lambda, t)\right) \\
& -a_{i-1}(t) \int_{-\infty}^{\infty} \lambda^{2 k} P_{i-1}(\lambda, t) P_{i+1}(\lambda, t) d \rho(\lambda, t)
\end{aligned}
$$

(for $k=1$ this formula coincides with Eq. (3.10)). From Eq. (3.12) the desired systems can be obtained in the same way as the system (3.2) was derived from Eq. (3.10). This procedure is easy programmable. In particular, we get the following systems $\left(V_{1}\right.$ is the initial system $\left.(3.2)\right)$ :

$$
\begin{aligned}
V_{2}: \dot{a}_{i} & =\frac{a_{i}}{2}\left(a_{i+1}^{2} \sum_{k=0}^{2} a_{i+k}^{2}-a_{i-1}^{2} \sum_{k=0}^{2} a_{i-k}^{2}\right), \\
V_{3}: \dot{a}_{i} & =\frac{a_{i}}{2}\left(a_{i+1}^{2} \sum_{k=0}^{2} a_{i+k}^{2} \sum_{p=0}^{k+1} a_{i+p}^{2}-a_{i-1}^{2} \sum_{k=0}^{2} a_{i-k}^{2} \sum_{p=0}^{k+1} a_{i-p}^{2}\right), \\
V_{4}: \dot{a}_{i} & =\frac{a_{i}}{2}\left(a_{i+1}^{2} \sum_{k=-1}^{2} a_{i+k}^{2} \sum_{p=0}^{k+1} a_{i+p}^{2} \sum_{q=r}^{p+1} a_{i+q}^{2}-a_{i-1}^{2} \sum_{k=-1}^{2} a_{i-k}^{2} \sum_{p=0}^{k+1} a_{i-p}^{2} \sum_{q=r}^{p+1} a_{i-q}^{2}\right), \\
\text { where } r & =\max (0,-k) ;
\end{aligned}
$$

Then it can be checked that each system $V_{k}$ satisfies the Lax equation

$$
\dot{L}(t)=\left[L(t), A_{k}(t)\right]
$$

where $A_{k}(t)=\operatorname{skew}\left(L^{2 k}(t) / 2\right)$ (cf. Eq. (3.4)). In other words, $V_{k}$ are the systems of Volterra (or KM in terms of [16]) hierarchy. Note that the procedure of obtaining $V_{k}$ from Eq. (3.12) is computationally faster than the one based on Eq. (3.14), because no matrix calculations are needed.

Also, the Volterra hierarchy can be generated via the bi-Hamiltonian structure of the initial system $\dot{b}_{i}=b_{i}\left(b_{i+1}-b_{i-1}\right)$, based on the two Poisson brackets [10]:

$$
\left\{b_{i+1}, b_{i}\right\}_{2}=b_{i} b_{i+1},\left\{b_{i+1}, b_{i}\right\}_{3}=b_{i} b_{i+1}\left(b_{i}+b_{i+1}\right), \quad\left\{b_{i+2}, b_{i}\right\}_{3}=b_{i} b_{i+1} b_{i+2} .
$$

Namely, for the systems of Volterra hierarchy $\left\{V_{k}\right\}_{k=1}^{\infty}$, written in coordinates $b_{k}$, we have

$$
V_{k}: \dot{b}_{i}=\left\{H_{k-1}, b_{i}\right\}_{3}=\left\{H_{k}, b_{i}\right\}_{2},
$$

where $H_{k}$ are the Hamiltonians, in particular, for $V_{1}: H_{0}=\sum_{i} \ln b_{i}, H_{1}=\sum_{i} b_{i}=\operatorname{Tr}\left(L^{2} / 2\right)$. This bi-Hamiltonian formalism, which dates back from the work of Magri [15], leads to an algorithm (offered in [16]) for generating the systems $V_{k}$ which can be represented by the diagram:

$$
H_{k-1} \stackrel{\{,\}_{3}}{\rightarrow} V_{k-1} \stackrel{\{,\}_{2}}{\rightarrow} H_{k} \stackrel{\{,\}_{3}}{\rightarrow} V_{k} \rightarrow \ldots
$$


(here we tacitly assume that $H_{k}$ are finite, which holds, e.g., for the finite or periodic lattices). In [16], $V_{2}$ and $V_{3}$ were obtained in this manner. In general, to obtain higher systems using this scheme, one should get the whole chain of "lower" Hamiltonians. However, as noted in [16], in this particular case all the Hamiltonians can be calculated independently by the formula

$$
H_{k}=\operatorname{Tr}\left(\frac{L^{2 k}}{2}\right)
$$

where $L(t)$ is defined by Eq. (3.3) (in the finite case $L(t)$ is a finite Jacobi matrix). The Hamiltonians of Volterra hierarchy are presented in Table 1, where

$$
\begin{aligned}
& H_{2}=\sum_{i}\left(\frac{a_{i}^{4}}{2}+a_{i}^{2} a_{i+1}^{2}\right)=\sum_{i}\left(\frac{b_{i}^{2}}{2}+b_{i} b_{i+1}\right), \\
& H_{3}=\sum_{i}\left(\frac{\left(b_{i+1}+b_{i}\right)^{3}-b_{i+1}^{3}}{3}+b_{i} b_{i+1} b_{i+2}\right), \\
& H_{4}=\sum_{i}\left(\frac{\left(b_{i+1}+b_{i}\right)^{4}-b_{i+1}^{4}}{4}+b_{i} b_{i+1} b_{i+2}\left(b_{i}+2 b_{i+1}+b_{i+2}+b_{i+3}\right)\right) .
\end{aligned}
$$

Table 1. Hamiltonians of Volterra hierarchy

\begin{tabular}{cccccc}
\hline System & $V_{1}$ & $V_{2}$ & $V_{3}$ & $V_{4}$ & $\ldots$ \\
\hline$\{,\}_{2}$ & $H_{1}$ & $H_{2}$ & $H_{3}$ & $H_{4}$ & $\ldots$ \\
$\{,\}_{3}$ & $H_{0}$ & $H_{1}$ & $H_{2}$ & $H_{3}$ & $\ldots$ \\
\hline
\end{tabular}

Although the proposed approach to generation of Volterra-type systems does not require the knowledge of these Hamiltonians, we can first derive $V_{k}$ from Eq. (3.12) and then find $H_{k}$ from the Hamiltonian representation of $V_{k}$ with respect to $\{,\}_{2}$. For example, to obtain $V_{5}$ given $V_{4}$, we can calculate $H_{4}$ in this manner and further on, applying $\{,\}_{3}$, find the equations for $V_{5}$ :

$$
\begin{aligned}
\dot{b}_{i} & =b_{i}\left(b_{i+1} \sum_{k=0}^{2} b_{i+k} \sum_{p=0}^{k+1} b_{i+p} \sum_{q=0}^{p+1} b_{i+q} \sum_{r=0}^{q+1} b_{i+r}-\right. \\
& -b_{i-1} \sum_{k=0}^{2} b_{i-k} \sum_{p=0}^{k+1} b_{i-p} \sum_{q=0}^{p+1} b_{i-q} \sum_{r=0}^{q+1} b_{i-r}+ \\
& +b_{i+1} \sum_{m=-2}^{-1} b_{i+m} \sum_{n=m+1}^{2 m+3} b_{i+n} \sum_{k=0}^{n+1} b_{i+k} \sum_{l=l 1}^{l 1+k(k-m)(1-|n|)} b_{i+l}- \\
& \left.-b_{i-1} \sum_{m=-2}^{-1} b_{i-m} \sum_{n=m+1}^{2 m+3} b_{i-n} \sum_{k=0}^{n+1} b_{i-k} \sum_{l=l 1}^{l 1+k(k-m)(1-|n|)} b_{i-l}\right),
\end{aligned}
$$

where $l 1=|k-n-m-2|+2\left\{\frac{k n m}{2}\right\}$. A straightforward application of Eq. (3.12) for $k=5$ (with the help from MATLAB Symbolic Toolbox) leads to the same result with the comparable computation time. 


\section{Nonsymmetric case}

\subsection{Volterra and Toda hierarchies}

First consider the infinite three-diagonal matrices of the form

$$
M=\left(\begin{array}{cccccc}
0 & 0 & 0 & 0 & 0 \\
1 & a_{1,1} & a_{1,2} & 0 & 0 \\
0 & 1 & a_{2,2} & a_{2,3} & 0 & \\
0 & \ddots & \ddots & \ddots & \ddots & \ddots
\end{array}\right), \quad a_{i, i}, a_{i, i+1} \in \mathbb{C}, \quad a_{i, i+1} \neq 0, \quad i \in \mathbb{N} .
$$

In the following we will identify each matrix $\mathrm{M}$ with the (possibly unbounded) nonsymmetric operator defined as the closure of the operator acting on the dense set of finite vectors from $l^{2}$, where the action of this operator is described via matrix calculus. In the same manner as above, we can define the Weyl function of $M$ by the formula $m(z)=\left(R_{z} e_{0}, e_{1}\right)$, where $R_{z}=(z E-M)^{-1}$ is the resolvent of $M$, its formal power expansion at infinity: $m_{\infty}(\lambda, L):=\sum_{k=0}^{\infty} \frac{S_{k}}{\lambda^{k+1}}, S_{k}=\left(M^{k}\right)_{1,0}$ $\left(S_{0}=0, S_{1}=1\right)$ and its moment sequence $S=\left\{S_{k}\right\}_{k=0}^{\infty}$. This moment sequence of the Weyl function generates the following bilinear mapping on the ring of polynomials: for $F(\lambda)=\sum_{p=0}^{i} F_{p} \lambda^{p}$, $G(\lambda)=\sum_{l=0}^{j} G_{l} \lambda^{l}$

$$
\left\langle F(\lambda), m_{\infty}(\lambda, L) G(\lambda)\right\rangle=\left\langle 1, F(\lambda) m_{\infty}(\lambda, L) G(\lambda)\right\rangle=\sum_{p=0}^{i} \sum_{l=0}^{j} F_{p} S_{p+l+1} G_{l} .
$$

Let $\Delta_{k}$ be determinants of the Hankel matrices $\left(S_{i+j+1}\right)_{i, j=0}^{k}, k \in \mathbb{Z}_{+}$. Similarly to Eqs. (2.6), the elements of $M$ can be found by the formulas

$$
a_{i, i+1}=\frac{\Delta_{i} \Delta_{i-2}}{\Delta_{i-1}^{2}}, \quad a_{i, i}=\frac{D_{i-1}}{\Delta_{i-1}}-\frac{D_{i-2}}{\Delta_{i-2}}
$$

where

$$
D_{i}=\operatorname{det}\left(\begin{array}{cccc}
S_{1} & \ldots & S_{i} & S_{i+2} \\
\vdots & & \vdots & \vdots \\
S_{i+1} & \ldots & S_{2 i} & S_{2 i+2}
\end{array}\right), \quad \Delta_{-1}=1, \quad D_{-1}=0, \quad D_{0}=S_{2}
$$

The following criterion for the sequence $S=\left\{S_{k}\right\}_{k=0}^{\infty}$ to be the moment sequence corresponding to the Weyl function of a certain matrix $M$ holds:

Theorem 3. The sequence $S$ is the moment sequence of the Weyl function of the matrix $M$ defined by Eq. (4.1) iff

- $S_{0}=0, S_{1}=1$ (normalization condition);

- for all $k \in \mathbb{Z}_{+}$,

$$
\Delta_{k} \neq 0
$$


The latter condition is equivalent to condition (2.1) for the Jacobi matrices.

This result follows from Theorem 2 of [17], which establishes a similar criterion for a wider class of infinite band matrices. As in the case of Jacoby matrices, the sparsity criterion holds for the matrices $M$ :

$$
\left\{a_{i, i}=0\right\}_{i=1}^{\infty} \Longleftrightarrow\left\{S_{2 k}=0\right\}_{k=0}^{\infty} .
$$

Now consider the Lax representation for the system (3.1) with the nonsymmetric matrix $L=L(t)$ :

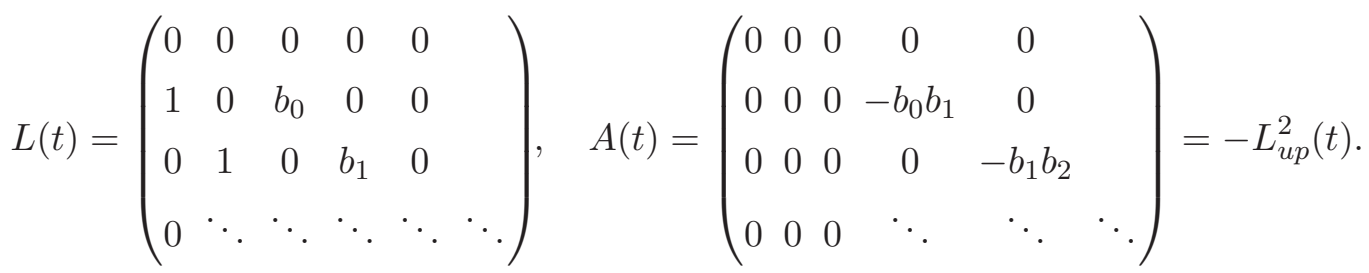

Also assume that $b_{i}(0) \neq 0, i \in \mathbb{Z}_{+}$. Obviously, $L(t)$ is a (sparse) matrix of the same structure as $M$, so all above results are applicable to this case.

For the matrix $L$ we define the polynomials $\left(P_{k}\right)_{k=0}^{\infty}, P_{k}=P_{k}(\lambda, t)$ satisfying the equation

$$
P_{k-1}+b_{k-1} P_{k+1}=\lambda y_{k}, \quad \lambda \in \mathbb{C}, \quad k=1,2, \ldots
$$

with the initial conditions $P_{0}=0, P_{1}=1$, and the polynomials $\left(T_{k}\right)_{k=0}^{\infty}, T_{k}=T_{k}(\lambda, t)$ satisfying the "adjoint" difference equation:

$$
T_{k-1} b_{k-2}+T_{k+1}=\lambda T_{k}, \quad k \in \mathbb{N}, \quad b_{-1}=1 ; \quad T_{0}=0, \quad T_{1}=1 .
$$

One can check (by induction) the orthogonality relations with respect to $m_{\infty}(\lambda, L, t)$ corresponding to $L(t)$ :

$$
\left\langle 1, P_{i}(\lambda, t) m_{\infty}(\lambda, L, t) T_{j}(\lambda, t)\right\rangle=\delta_{i, j}, \quad i, j \in \mathbb{N} .
$$

Therefore, we get

$$
b_{k}=\left\langle 1, \lambda P_{k+1}(\lambda, t) m_{\infty}(\lambda, L, t) T_{k+2}(\lambda, t)\right\rangle .
$$

Using the equation $\left(L^{k}(t)\right)^{\cdot}=L^{k} A-A L^{k}$, which follows from the Lax representation, we find the evolutionary equations for the moments $S_{k}$ :

$$
\dot{S}_{k}=S_{k+2}-S_{3} S_{k}, \quad k \in \mathbb{Z}_{+},
$$

from which it follows that

$$
\left\langle 1, \lambda P_{k+1}(\lambda, t) \dot{m}_{\infty}(\lambda, L, t) T_{k+2}(\lambda, t)\right\rangle=\left\langle 1, \lambda^{3} P_{k+1}(\lambda, t) m_{\infty}(\lambda, L, t) T_{k+2}(\lambda, t)\right\rangle-S_{3} b_{k} .
$$

Thus, as in the proof of Theorem 1, we are coming to the equation which is equivalent to that of the Volterra lattice (3.1) (cf. Eq. (3.10)):

$$
\begin{aligned}
\dot{b}_{k} & =\left\langle 1, \lambda^{3} P_{k+1}(\lambda, t) m_{\infty}(\lambda, L, t) T_{k+2}(\lambda, t)\right\rangle \\
& -\left\langle 1, \lambda^{2} P_{k+1}(\lambda, t) m_{\infty}(\lambda, L, t) T_{k+1}(\lambda, t)\right\rangle b_{k}- \\
& -\left\langle 1, \lambda^{2} P_{k}(\lambda, t) m_{\infty}(\lambda, L, t) T_{k+2}(\lambda, t)\right\rangle .
\end{aligned}
$$

Similarly, we get the equations for the moments of another systems of Volterra hierarchy:

$$
V_{m}: \quad \dot{S}_{k}=S_{k+2 m}-S_{2 m+1} S_{k}, \quad m=1,2, \ldots
$$


and the equations for $V_{m}$, similar to Eqs. (3.12):

$$
\begin{aligned}
\dot{b}_{k} & =\left\langle 1, \lambda^{2 m+1} P_{k+1}(\lambda, t) m_{\infty}(\lambda, L, t) T_{k+2}(\lambda, t)\right\rangle- \\
& -\left\langle 1, \lambda^{2 m} P_{k+1}(\lambda, t) m_{\infty}(\lambda, L, t) T_{k+1}(\lambda, t)\right\rangle b_{k}- \\
& -\left\langle 1, \lambda^{2 m} P_{k}(\lambda, t) m_{\infty}(\lambda, L, t) T_{k+2}(\lambda, t)\right\rangle .
\end{aligned}
$$

Here we mention that unlike in the symmetric case, where the uniform boundedness of initial data guarantees the existence of a global solution of the corresponding Volterra system, here we can claim that for the bounded initial data the solution exists and is unique only on the interval $[0, \delta)$ for some $\delta>0$, see [14] for details.

Also note that the above formulas are valid in the non-Abelian case, e.g., when $b_{k}$ are matrices or operators (details of the inverse problem method applicable to this case can be found in [12]). For example, using Eqs. (4.8), we can derive equations for $V_{3}$ both in the Abelian and non-Abelian case (cf. the third of Eqs. (3.13)):

$$
\begin{aligned}
\dot{b}_{k} & =b_{k} b_{k+1} b_{k+2} b_{k+1}+b_{k} b_{k+1} b_{k+2} b_{k+2}+b_{k} b_{k+1} b_{k+2} b_{k+3}+b_{k} b_{k} b_{k} b_{k+1}+ \\
& +b_{k} b_{k+1} b_{k} b_{k+1}+b_{k} b_{k} b_{k+1} b_{k+1}+b_{k} b_{k} b_{k+1} b_{k+2}+b_{k} b_{k+1} b_{k+1} b_{k+1}+ \\
& +b_{k} b_{k+1} b_{k+1} b_{k+2}-b_{k-1} b_{k-2} b_{k-1} b_{k}-b_{k-2} b_{k-2} b_{k-1} b_{k}-b_{k-3} b_{k-2} b_{k-1} b_{k}- \\
& -b_{k-1} b_{k} b_{k} b_{k}-b_{k-1} b_{k} b_{k-1} b_{k}-b_{k-1} b_{k-1} b_{k} b_{k}-b_{k-2} b_{k-1} b_{k} b_{k}- \\
& -b_{k-1} b_{k-1} b_{k-1} b_{k}-b_{k-2} b_{k-1} b_{k-1} b_{k}+b_{k} b_{k-1} b_{k} b_{k+1}-b_{k-1} b_{k} b_{k+1} b_{k} .
\end{aligned}
$$

Also, consider the Toda lattice $T o_{1}$ :

$$
\dot{A}_{k}=A_{k} B_{k+1}-B_{k} A_{k}, \quad \dot{B}_{k}=A_{k}-A_{k-1},
$$

where $A_{k}, B_{k} \in \mathbb{C}$ and $A_{k} \neq 0$ (the non-Abelian $T o_{1}$ can be written similarly). In the semiinfinite case it admits the Lax representation with the following infinite matrices $\widetilde{L}$ and $A$ :

$$
\begin{aligned}
& \widetilde{L}=\widetilde{L}(t)=\left(\begin{array}{cccccc}
0 & 0 & 0 & 0 & 0 & \\
1 & B_{0} & A_{0} & 0 & 0 & \\
0 & 1 & B_{1} & A_{1} & 0 & \\
0 & \ddots & \ddots & \ddots & \ddots & \ddots
\end{array}\right), \\
& A=A(t)=\left(\begin{array}{cccccc}
0 & 0 & 0 & 0 & \\
0 & 0 & -A_{0} & 0 & \\
0 & 0 & 0 & -A_{1} & \\
0 & 0 & \ddots & \ddots & \ddots
\end{array}\right)=-\widetilde{L}_{u p}(t) .
\end{aligned}
$$

Similarly as above, define $\left(P_{k}(\lambda, t)\right)_{k=0}^{\infty}$ as the polynomials satisfying

$$
P_{k-1}+B_{k-1} P_{k}+A_{k-1} P_{k+1}=\lambda P_{k}, \quad k=1,2, \ldots ; \quad P_{0}=0, \quad P_{1}=1 ;
$$

and the "adjoint" polynomials $\left(T_{k}(\lambda, t)\right)_{k=0}^{\infty}$ as the ones satisfying the equation

$$
T_{k-1} A_{k-2}+T_{k} B_{k-1}+T_{k+1}=\lambda T_{k}, \quad k=1,2, \ldots ; \quad A_{-1}=1 ; \quad T_{0}=0, \quad T_{1}=1 .
$$


These two polynomial systems are mutually orthogonal with respect to $m_{\infty}(\lambda, \widetilde{L}, t)$, e.g., the relations similar to Eq. (4.5) hold for them. For $T o_{1}$, the equation for the moments similar to Eq. (4.6) for the Volterra system takes the form

$$
\dot{\widetilde{S}}_{k}=\widetilde{S}_{k+1}-B_{0}(t) \widetilde{S}_{k}=\widetilde{S}_{k+1}-\widetilde{S}_{2} \widetilde{S}_{k}, \quad \widetilde{S}_{k}=\left(\widetilde{L}^{k}(t)\right)_{1,0} .
$$

Using Eq. (4.11), one may check that Eqs. (4.9) can be written as

$$
\begin{aligned}
\dot{A}_{k} & =\left\langle 1, \lambda^{2} P_{k+1}(\lambda, t) m_{\infty}(\lambda, \widetilde{L}, t) T_{k+2}(\lambda, t)\right\rangle- \\
& -\left\langle 1, \lambda P_{k+1}(\lambda, t) m_{\infty}(\lambda, \widetilde{L}, t) T_{k+1}(\lambda, t)\right\rangle A_{k}- \\
& -B_{k}\left\langle 1, \lambda P_{k+1}(\lambda, t) m_{\infty}(\lambda, \widetilde{L}, t) T_{k+2}(\lambda, t)\right\rangle \\
& -\left\langle 1, \lambda P_{k}(\lambda, t) m_{\infty}(\lambda, \widetilde{L}, t) T_{k+2}(\lambda, t)\right\rangle ; \\
\dot{B}_{k} & =\left\langle 1, \lambda^{2} P_{k+1}(\lambda, t) m_{\infty}(\lambda, \widetilde{L}, t) T_{k+1}(\lambda, t)\right\rangle- \\
& -\left\langle 1, \lambda P_{k+1}(\lambda, t) m_{\infty}(\lambda, \widetilde{L}, t) T_{k}(\lambda, t)\right\rangle A_{k-1}- \\
& -\left\langle 1, \lambda P_{k+1}(\lambda, t) m_{\infty}(\lambda, \widetilde{L}, t) T_{k+1}(\lambda, t)\right\rangle B_{k}- \\
& -\left\langle 1, \lambda P_{k}(\lambda, t) m_{\infty}(\lambda, \widetilde{L}, t) T_{k+1}(\lambda, t)\right\rangle .
\end{aligned}
$$

The last term in the equation for $\dot{A}_{k}$ is actually zero, but we write it here for convenience to unify the shape of all equations of Toda hierarchy $\left\{T o_{m}\right\}_{m=1}^{\infty}$. Namely, the evolutionary equations for the moments of the elements $T o_{m}$ of this hierarchy are written as

$$
\dot{\widetilde{S}}_{k}=\widetilde{S}_{k+m}-\widetilde{S}_{1+m} \widetilde{S}_{k}, \quad m=1,2, \ldots,
$$

using which, we obtain

$$
\begin{aligned}
& T o_{m}: \\
& \dot{A}_{k}=\left\langle 1, \lambda^{m+1} P_{k+1, t}(\lambda, t) m_{\infty}(\lambda, \widetilde{L}, t) T_{k+2}(\lambda, t)\right\rangle- \\
&-\left\langle 1, \lambda^{m} P_{k+1}(\lambda, t) m_{\infty}(\lambda, \widetilde{L}, t) T_{k+1}(\lambda, t)\right\rangle A_{k}- \\
&- B_{k}\left\langle 1, \lambda^{m} P_{k+1}(\lambda, t) m_{\infty}(\lambda, \widetilde{L}, t) T_{k+2}(\lambda, t)\right\rangle \\
&-\left\langle 1, \lambda^{m} P_{k}(\lambda, t) m_{\infty}(\lambda, \widetilde{L}, t) T_{k+2}(\lambda, t)\right\rangle ; \\
& \dot{B}_{k}=\left\langle 1, \lambda^{m+1} P_{k+1}(\lambda, t) m_{\infty}(\lambda, \widetilde{L}, t) T_{k+1}(\lambda, t)\right\rangle- \\
&-\left\langle 1, \lambda^{m} P_{k+1}(\lambda, t) m_{\infty}(\lambda, \widetilde{L}, t) T_{k}(\lambda, t)\right\rangle A_{k-1}- \\
&-\left\langle 1, \lambda^{m} P_{k+1}(\lambda, t) m_{\infty}(\lambda, \widetilde{L}, t) T_{k+1}(\lambda, t)\right\rangle B_{k}- \\
&-\left\langle 1, \lambda^{m} P_{k}(\lambda, t) m_{\infty}(\lambda, \widetilde{L}, t) T_{k+1}(\lambda, t)\right\rangle .
\end{aligned}
$$

One can check that the systems defined by Eqs. (4.13) satisfy the Lax equations $\dot{\widetilde{L}}(t)=$ $=\left[\widetilde{L}(t), A_{m}(t)\right]$, where $\widetilde{L}(t)$ is defined by Eq. (4.10) and $A_{m}(t)=-\widetilde{L}_{u p}^{m}(t)$.

Theorem 4. The equations of Volterra hierarchy (4.8) can be transformed to their Toda counterparts defined by Eqs. (4.13). The corresponding mapping for the moments is defined as

$$
S_{2 k-1} \rightarrow \widetilde{S}_{k}, \quad k \in \mathbb{N}, \quad \widetilde{S}_{0}=0,
$$

which implies

$$
B_{k}=b_{2 k}+b_{2 k-1}, \quad A_{k}=b_{2 k} b_{2 k+1} ; \quad k \in \mathbb{Z}_{+} ; \quad b_{-1}=0 .
$$


Proof. Let $S=\left\{S_{k}\right\}_{k=0}^{\infty}$ be the moment sequence corresponding to $V_{m}$. Then, according to (4.14), we get

$$
\dot{\widetilde{S}}_{k}=\dot{S}_{2 k-1} \stackrel{b y}{\stackrel{(4.8)}{=}} S_{2 k+2 m-1}-S_{2 m+1} S_{2 k-1}=\widetilde{S}_{k+m}-\widetilde{S}_{m+1} \widetilde{S}_{k},
$$

which is exactly Eqs. (4.12), so we can derive from them Eqs. (4.13).

To establish Eqs. (4.15), we first find from Eqs. (4.2) that

$$
b_{k}=\frac{\Delta_{k+1} \Delta_{k-1}}{\Delta_{k}^{2}}, \text { where } \Delta_{k}=\operatorname{det}\left(\begin{array}{cccccc}
S_{1} & 0 & S_{3} & 0 & \ldots & S_{k+1} \\
0 & S_{3} & 0 & S_{5} & \ldots & S_{k+2} \\
S_{3} & 0 & S_{5} & 0 & \ldots & S_{k+3} \\
0 & S_{5} & 0 & S_{7} & \ldots & S_{k+4} \\
\vdots & \vdots & \vdots & & & \vdots \\
S_{k+1} & S_{k+2} & S_{k+3} & \ldots & S_{2 k} & S_{2 k+1}
\end{array}\right)
$$

$\left(S_{k}=L_{1,0}^{k}\right)$. Using the sparse structure of $\Delta_{k}$, we get the following factorization of the latter (can be proved by direct check):

$$
\Delta_{k}=L_{m} R_{m^{\prime}}, \quad k \in \mathbb{N}
$$

where

$$
\begin{aligned}
L_{m}=\left|\begin{array}{cccc}
S_{1} & S_{3} & \ldots & S_{2 m+1} \\
S_{3} & S_{5} & \ldots & S_{2 m+3} \\
\vdots & \vdots & \vdots & \vdots \\
S_{2 m+1} & S_{2 m+3} & \ldots & S_{4 m+1}
\end{array}\right|, \quad R_{m^{\prime}}=\left|\begin{array}{cccc}
S_{3} & S_{5} & \ldots & S_{2 m^{\prime}+1} \\
S_{5} & S_{7} & \ldots & S_{2 m^{\prime}+3} \\
\vdots & \vdots & \vdots & \vdots \\
S_{2 m^{\prime}+1} & S_{2 m^{\prime}+3} & \ldots & S_{4 m^{\prime}-1}
\end{array}\right| \\
m=\left[\frac{k}{2}\right], \quad m^{\prime}=\left[\frac{k-1}{2}\right]+1 .
\end{aligned}
$$

Set $\widetilde{\Delta}_{l}=\operatorname{det}\left(\widetilde{H}_{l}\right)$ where $\widetilde{H}_{l}=\left\{\widetilde{S}_{i+j+1}\right\}_{i, j=0}^{l}$. According to (4.14), $\widetilde{\Delta}_{l}=\operatorname{det}\left(\left\{S_{2(i+j)+1}\right\}_{i, j=0}^{l}\right)$ and therefore, $\widetilde{\Delta}_{l}=L_{l}$ for $l \in \mathbb{N}$. By Theorem $3, \Delta_{k} \neq 0$ for all $k$, and it follows from formula (4.16) that $\widetilde{\Delta}_{l} \neq 0$ for all $l$ as well; therefore, by the same theorem, $\widetilde{S}$ is the moment sequence corresponding to a certain matrix $M$. If we denote in this matrix $a_{k+1, k+2}$ and $a_{k+1, k+1}$, $k \in \mathbb{Z}_{+}$, by $A_{k}$ and $B_{k}$, respectively, then it follows from Eqs. (4.2) that

$$
A_{k}=\frac{\widetilde{\Delta}_{k+1} \widetilde{\Delta}_{k-1}}{\widetilde{\Delta}_{k}^{2}}, \quad B_{k}=\frac{\widetilde{D}_{k}}{\widetilde{\Delta}_{k}}-\frac{\widetilde{D}_{k-1}}{\widetilde{\Delta}_{k-1}}, \quad \widetilde{D}_{k}=\left|\begin{array}{cccc}
\widetilde{S}_{1} & \ldots & \widetilde{S}_{k} & \widetilde{S}_{k+2} \\
\vdots & & \vdots & \vdots \\
\widetilde{S}_{k+1} & \ldots & \widetilde{S}_{2 k} & \widetilde{S}_{2 k+2}
\end{array}\right|,
$$

$\left(\widetilde{D}_{0}=\widetilde{S}_{2}, \widetilde{D}_{-1}=0, \widetilde{\Delta}_{-1}=1\right)$. Also note that according to $(4.14)$,

We have

$$
R_{k}=\left|\begin{array}{cccc}
\widetilde{S}_{2} & \widetilde{S}_{3} & \ldots & \widetilde{S}_{k+1} \\
\vdots & \vdots & & \vdots \\
\widetilde{S}_{k+1} & \widetilde{S}_{k+2} & \cdots & \widetilde{S}_{2 k}
\end{array}\right|
$$

$$
A_{0}=\frac{\widetilde{\Delta}_{1}}{\widetilde{\Delta}_{0}^{2}}=\left|\begin{array}{ll}
S_{1} & S_{3} \\
S_{3} & S_{5}
\end{array}\right|=b_{0}^{2}+b_{0} b_{1}-b_{0}^{2}=b_{0} b_{1} .
$$


For $k>1$

$$
b_{2 k} b_{2 k+1}=\frac{\Delta_{2 k-1} \Delta_{2 k+2}}{\Delta_{2 k} \Delta_{2 k+1}}=\frac{L_{k-1} R_{k} L_{k+1} R_{k+1}}{L_{k} R_{k} L_{k} R_{k+1}}=\frac{L_{k+1} L_{k-1}}{L_{k}^{2}}=\frac{\widetilde{\Delta}_{k+1} \widetilde{\Delta}_{k-1}}{\widetilde{\Delta}_{k}^{2}}=A_{k} .
$$

The formula for $B_{k}$ follows from the identity

$$
\frac{\widetilde{D}_{l}}{\widetilde{\Delta}_{l}}=\sum_{m=0}^{2 l} b_{m}, l \geqslant 0
$$

It can be proved by induction on $l$. For $l=0, \frac{\widetilde{D}_{0}}{\widetilde{\Delta}_{0}}=\widetilde{S}_{2}=S_{3}=b_{0}$. Suppose the identity defined by formula (4.17) is proved for $l=0, \ldots, k-1$. It means that

$$
\begin{aligned}
\sum_{m=0}^{2 k} b_{k} & =\frac{\widetilde{D}_{k-1}}{\widetilde{\Delta}_{k-1}}+b_{2 k-1}+b_{2 k}=\frac{\widetilde{D}_{k-1}}{\widetilde{\Delta}_{k-1}}+\frac{\widetilde{\Delta}_{k} R_{k-1}}{\widetilde{\Delta}_{k-1} R_{k}}+\frac{\widetilde{\Delta}_{k-1} R_{k+1}}{\widetilde{\Delta}_{k} R_{k}}= \\
& =\frac{\widetilde{\Delta}_{k}\left(\widetilde{D}_{k-1} R_{k}+\widetilde{\Delta}_{k} R_{k-1}\right)+\widetilde{\Delta}_{k-1}^{2} R_{k+1}}{\widetilde{\Delta}_{k-1} \widetilde{\Delta}_{k} R_{k}} .
\end{aligned}
$$

For the expression in brackets the following representation holds:

$$
\widetilde{\Delta}_{k} R_{k-1}+\widetilde{D}_{k-1} R_{k}=\widetilde{\Delta}_{k-1} \widetilde{D}_{k-1}^{(1)} \text {, where } \widetilde{D}_{k}^{(1)}=\left|\begin{array}{cccc}
\widetilde{S}_{2} & \ldots & \widetilde{S}_{k+1} & \widetilde{S}_{k+3} \\
\vdots & & \vdots & \vdots \\
\widetilde{S}_{k+2} & \ldots & \widetilde{S}_{2 k+1} & \widetilde{S}_{2 k+3}
\end{array}\right| .
$$

It follows from one general identity for determinants proved in the Appendix. To establish the identity (4.17) for $l=k$, we have to check that

$$
\widetilde{\Delta}_{k} \widetilde{D}_{k-1}^{(1)}+\widetilde{\Delta}_{k-1} R_{k+1}=\widetilde{D}_{k} R_{k}
$$

or

$$
\begin{gathered}
\left|\begin{array}{cccc}
\widetilde{S}_{1} & \widetilde{S}_{2} & \ldots & \widetilde{S}_{k+1} \\
\widetilde{S}_{2} & \widetilde{S}_{3} & \ldots & \widetilde{S}_{k+2} \\
\vdots & \vdots & & \vdots \\
\widetilde{S}_{k+1} & \widetilde{S}_{k+2} & \ldots & \widetilde{S}_{2 k+1}
\end{array}\right|\left|\begin{array}{ccccc}
\widetilde{S}_{2} & \widetilde{S}_{3} & \ldots & \widetilde{S}_{k} & \widetilde{S}_{k+2} \\
\widetilde{S}_{3} & \widetilde{S}_{4} & \ldots & \widetilde{S}_{k+1} & \widetilde{S}_{k+3} \\
\vdots & \vdots & & \vdots & \vdots \\
\widetilde{S}_{k+1} & \widetilde{S}_{k+2} & \ldots & \widetilde{S}_{2 k-1} & \widetilde{S}_{2 k+1}
\end{array}\right|+ \\
+\left|\begin{array}{ccccc}
\widetilde{S}_{1} & \widetilde{S}_{2} & \ldots & \widetilde{S}_{k} \\
\widetilde{S}_{2} & \widetilde{S}_{3} & \ldots & \widetilde{S}_{k+1} \\
\vdots & \vdots & & \vdots \\
\widetilde{S}_{k} & \widetilde{S}_{k+1} & \ldots & \widetilde{S}_{2 k-1}
\end{array}\right|\left|\begin{array}{cccc}
\widetilde{S}_{2} & \widetilde{S}_{3} & \ldots & \widetilde{S}_{k+2} \\
\widetilde{S}_{3} & \widetilde{S}_{4} & \ldots & \widetilde{S}_{k+3} \\
\vdots & \vdots & & \vdots \\
\widetilde{S}_{k+2} & \widetilde{S}_{k+3} & \ldots & \widetilde{S}_{2 k+2}
\end{array}\right|= \\
=\left|\begin{array}{ccccc}
\widetilde{S}_{1} & \ldots & \widetilde{S}_{k} & \widetilde{S}_{k+2} \\
\widetilde{S}_{2} & \ldots & \widetilde{S}_{k+1} & \widetilde{S}_{k+3} \\
\vdots & & \vdots & \vdots \\
\widetilde{S}_{2} & \widetilde{S}_{3} & \ldots & \widetilde{S}_{k+1} \\
\widetilde{S}_{3} & \widetilde{S}_{4} & \ldots & \widetilde{S}_{k+2} \\
\vdots & \vdots & & \vdots \\
\widetilde{S}_{k} & \ldots & \widetilde{S}_{2 k} & \widetilde{S}_{2 k+2}
\end{array}\right|\left|\begin{array}{cccc}
\widetilde{S}_{k+1} & \widetilde{S}_{k+2} & \ldots & \widetilde{S}_{2 k}
\end{array}\right| .
\end{gathered}
$$


The latter formula can be written as

$$
\left|\begin{array}{ccccccccc}
\widetilde{S}_{1} & \overbrace{}^{0} & \ldots & 0 & \widetilde{S}_{2} & \widetilde{S}_{3} & \ldots & \widetilde{S}_{k+1} & \widetilde{S}_{k+2} \\
\widetilde{S}_{2} & 0 & \ldots & 0 & \widetilde{S}_{3} & \widetilde{S}_{4} & \ldots & \widetilde{S}_{k+2} & \widetilde{S}_{k+3} \\
\vdots & \vdots & \vdots & \vdots & \vdots & \vdots & \ldots & \vdots & \vdots \\
\widetilde{S}_{k} & 0 & \ldots & 0 & \widetilde{S}_{k+1} & \widetilde{S}_{k+2} & \ldots & \widetilde{S}_{2 k} & \widetilde{S}_{2 k+1} \\
\widetilde{S}_{1} & \widetilde{S}_{2} & \ldots & \widetilde{S}_{k} & 0 & \ldots & 0 & \widetilde{S}_{k+1} & \widetilde{S}_{k+2} \\
\widetilde{S}_{2} & \widetilde{S}_{3} & \ldots & \widetilde{S}_{k+1} & 0 & \ldots & 0 & \widetilde{S}_{k+2} & \widetilde{S}_{k+3} \\
\vdots & \vdots & \vdots & \vdots & \vdots & \ldots & \vdots & \vdots & \vdots \\
\widetilde{S}_{k+1} & \widetilde{S}_{k+2} & \ldots & \widetilde{S}_{2 k} & 0 & \ldots & 0 & \widetilde{S}_{2 k+1} & \widetilde{S}_{2 k+2}
\end{array}\right|=0
$$

Using elementary matrix transformations, we can write this determinant as

$$
\left|\begin{array}{ccccccccc}
\widetilde{S}_{1} & \overbrace{}^{0} & \ldots & 0 & \widetilde{S}_{2} & \widetilde{S}_{3} & \ldots & \widetilde{S}_{k+1} & \widetilde{S}_{k+2} \\
\widetilde{S}_{2} & 0 & \ldots & 0 & \widetilde{S}_{3} & \widetilde{S}_{4} & \ldots & \widetilde{S}_{k+2} & \widetilde{S}_{k+3} \\
\vdots & \vdots & \vdots & \vdots & \vdots & \vdots & \ldots & \vdots & \vdots \\
\widetilde{S}_{k} & 0 & \ldots & 0 & \widetilde{S}_{k+1} & \widetilde{S}_{k+2} & \ldots & \widetilde{S}_{2 k} & \widetilde{S}_{2 k+1} \\
0 & \widetilde{S}_{2} & \ldots & \widetilde{S}_{k} & 0 & 0 & \ldots & \ldots & 0 \\
0 & \widetilde{S}_{3} & \ldots & \widetilde{S}_{k+1} & 0 & 0 & \ldots & \ldots & 0 \\
\vdots & \vdots & \ldots & \vdots & \vdots & \vdots & \ldots & \ldots & \vdots \\
0 & \widetilde{S}_{k+1} & \ldots & \widetilde{S}_{2 k-1} & 0 & 0 & \ldots & \ldots & 0 \\
\widetilde{S}_{k+1} & \widetilde{S}_{k+2} & \ldots & \widetilde{S}_{2 k} & \widetilde{S}_{k+2} & \widetilde{S}_{k+3} & \ldots & \widetilde{S}_{2 k+1} & \widetilde{S}_{2 k+2}
\end{array}\right|
$$

Expanding the determinant to the last row and applying the Laplace formula to the minors (of order $2 k$ ) appearing after the expansion, we get zero. Thus, the identity (4.17) is proved for all $l$.

\subsection{Finite case}

Now consider the finite systems $V_{m}$ and $T o_{m}$. In this case, the matrices $L(t)$ and $A(t)$ in the corresponding Lax representations are finite, so we first need to establish a "finite" version of Theorem 3. Namely, for $N \geqslant 2$ consider the matrix

$$
M_{N}=\left(\begin{array}{ccccc}
0 & 0 & 0 & 0 & 0 \\
1 & a_{1,1} & a_{1,2} & 0 & 0 \\
0 & 1 & a_{2,2} & a_{2,3} & 0 \\
0 & \ddots & \ddots & \ddots & \ddots \\
0 & \ldots & 0 & 1 & a_{N, N}
\end{array}\right), \quad a_{i, i}, a_{i, i+1} \in \mathbb{C}, \quad a_{i, i+1} \neq 0 .
$$

For $M_{N}$ we define its Weyl function $m_{N}(z)$ and the moment sequence $S$ of the latter, similarly as for the matrix $M$. Then the following statement holds. 
Theorem 5. The sequence $S$ is the moment sequence of the Weyl function $m_{N}(z)$ of the matrix $M_{N}$ iff

- $S_{0}=0, S_{1}=1$;

- $\Delta_{k} \neq 0$, for $k=0, \ldots, N-1$.

- there exist $C_{0}, \ldots, C_{N-1} \in \mathbb{C}$, such that

$$
S_{i}=\sum_{j=0}^{N-1} C_{j} S_{i-1-j}, \quad i=N+1, \ldots, \infty .
$$

The proof of this theorem for a wider class of finite band matrices is contained in [13]. It is proved in a similar manner to Theorem 3, and the proof relies on the fact that an infinite Hankel matrix $H=\left(S_{i+j+1}\right)_{i, j=0}^{\infty}$ has a finite rank $N$ if and only if the condition defined by Eq. (4.19) is fulfilled (see [18, Chapter 15]). In the finite case $m_{N}(z)$ is a rational function.

Now assume that we have a finite dynamical system which admits a Lax representation with the matrix $L=M_{N}$. Then it often happens that the quantities $C_{j}$ are the first integrals of the system. To be more precise, the following proposition holds.

Proposition 2. Assume that a dynamical system $F$ can be written as $\dot{M}_{N}=\left[M_{N}, A\right]$, where $M_{N}$ is defined by formula (4.18) and the moments of $m_{N}(z)$ satisfy the equations

$$
\dot{S}_{k}=f(t)\left(\sum_{l=1}^{p} d_{l} S_{k+l}-b(t) S_{k}\right), \quad k \in \mathbb{N},
$$

for some $f(t), b(t), d_{j} \in \mathbb{C}, p \in \mathbb{N}$. Then

$$
\dot{C}_{j}=0, \quad j=0, \ldots, N-1 .
$$

Proof. According to Eq. (4.20),

$$
\dot{S}_{N+1}=f(t)\left(\sum_{l=1}^{p} d_{l} S_{N+1+l}-b(t) S_{N+1}\right) .
$$

At the same time, it follows from Eq.(4.19) that

$$
\dot{S}_{N+1}=\sum_{j=0}^{N-1} C_{j} \dot{S}_{N-j}+\sum_{j=0}^{N-1} \dot{C}_{j} S_{N-j} .
$$

Applying Eq. (4.20) to $\dot{S}_{1}, \ldots, \dot{S}_{N}$ in the latter formula and using Eq. (4.19), we find

$$
\dot{S}_{N+1}=f(t)\left(\sum_{l=1}^{p} d_{l} S_{N+1+l}-b(t) S_{N+1}\right)+\sum_{j=0}^{N-1} \dot{C}_{j} S_{N-j} .
$$

Thus,

$$
\sum_{j=0}^{N-1} \dot{C}_{j} S_{N-j}=0
$$


Acting similarly with $\dot{S}_{N+2}, \ldots, \dot{S}_{2 N-1}$, we arrive at the system

$$
\left\{\begin{array}{l}
\dot{C}_{N-1} S_{1}+\ldots+\dot{C}_{0} S_{N}=0 \\
\vdots \\
\dot{C}_{N-1} S_{N}+\ldots+\dot{C}_{0} S_{2 N-1}=0
\end{array}\right.
$$

According to Theorem 5 , its determinant $\Delta_{N-1} \neq 0$, and the claim follows immediately.

As we see, Eqs. (4.7) and (4.12) are special cases of Eq. (4.20). Therefore, for the finite systems of Volterra and Toda hierarchy, the corresponding quantities $C_{j}$ are their first integrals (the same is true for the finite non-Abelian Toda and Volterra lattices, see [19] for details).

Using the Cartan-Weyl classification of semisimple Lie algebras, Bogoyavlenskij (see, e.g., [9]) found the finite integrable systems of Volterra and Toda type, related to each class of algebras. In his terminology, the above Volterra and Toda lattices in the finite case are the $A_{n}$-Volterra and Toda systems. Using Theorem 4, one may check that the mapping defined by Eqs. (4.14)-(4.15) transforms the $A_{n}$ Volterra systems (for $n \geqslant 2$ ) into their $A_{m}$ Toda counterparts, where $m=\lfloor n / 2\rfloor$. As an example, consider the $A_{3}$ Volterra system:

$$
\dot{b}_{0}=b_{0} b_{1}, \quad \dot{b}_{1}=b_{1}\left(b_{2}-b_{0}\right), \quad \dot{b}_{2}=-b_{2} b_{1} .
$$

It admits the Lax representation (4.4) with the following matrix $L(t)$ :

$$
L=\left(\begin{array}{ccccc}
0 & 0 & 0 & 0 & 0 \\
1 & 0 & b_{0} & 0 & 0 \\
0 & 1 & 0 & b_{1} & 0 \\
0 & 0 & 1 & 0 & b_{2} \\
0 & 0 & 0 & 1 & 0
\end{array}\right) .
$$

From Theorem 5 we find that for the moments of its Weyl function the following relations hold:

$$
S_{i}=\sum_{j=0}^{4} C_{j} S_{i-1-j}=\left(b_{0}+b_{1}+b_{2}\right) S_{i-2}+\left(b_{0} b_{2}\right) S_{i-4}, \quad i \geqslant 5 .
$$

By Proposition $2,\left(b_{0}+b_{1}+b_{2}\right)$ and $b_{0} b_{2}$ are the first integrals of the system. Now, applying Eqs. (4.14)-(4.15), we transform Eqs. (4.22) into the Toda $A_{1}$ system with the matrix $\widetilde{L}(t)$ :

$$
\widetilde{L}=\left(\begin{array}{ccc}
0 & 0 & 0 \\
1 & B_{0} & A_{0} \\
0 & 1 & B_{1}
\end{array}\right)=\left(\begin{array}{ccc}
0 & 0 & 0 \\
1 & b_{0} & b_{0} b_{1} \\
0 & 1 & b_{1}+b_{2}
\end{array}\right)
$$

and the quantities $\left(b_{0}+b_{1}+b_{2}\right)=\operatorname{tr}(\widetilde{L})$ and $b_{0} b_{2}$ are the first integrals of the transformed system.

Also, consider the following Volterra system $B_{3}[11]$ from the class $B_{n}$ :

$$
\dot{b}_{0}=b_{0} b_{1}, \quad \dot{b}_{1}=b_{1}\left(b_{2}-b_{0}\right), \quad \dot{b}_{2}=-b_{2} b_{1}-b_{2}^{2} .
$$


As noted in [11], the equations for $B_{3}$ can be obtained from the equations of Volterra system $A_{6}$ with the following Lax matrix $\widehat{L}$ :

$$
\widehat{L}(t)=\left(\begin{array}{cccccccc}
0 & 0 & 0 & 0 & 0 & 0 & 0 & 0 \\
1 & 0 & b_{0} & 0 & 0 & 0 & 0 & 0 \\
0 & 1 & 0 & b_{1} & 0 & 0 & 0 & 0 \\
0 & 0 & 1 & 0 & b_{2} & 0 & 0 & 0 \\
0 & 0 & 0 & 1 & 0 & -b_{2} & 0 & 0 \\
0 & 0 & 0 & 0 & 1 & 0 & -b_{1} & 0 \\
0 & 0 & 0 & 0 & 0 & 1 & 0 & -b_{0} \\
0 & 0 & 0 & 0 & 0 & 0 & 1 & 0
\end{array}\right) .
$$

Again, applying Theorem 5, we find that

$$
S_{i}=C_{3} S_{i-4}=\left(b_{0}^{2}+b_{1}^{2}+b_{0} b_{1}+2 b_{1} b_{2}\right) S_{i-4}, \quad i \geqslant 8
$$

(the other $C_{j}$ here are equal to zero). Thus, $C_{3}$ is the first integral of the system $A_{6}$ (this is a known fact, since one can check that $\left.C_{3}=\operatorname{tr}\left(\widehat{L}^{4}\right) / 4\right)$ and, therefore, of $B_{3}$.

As to the transformations between Volterra and Toda systems belonging to the classes other than $A_{n}$ and $B_{n}$, their detailed study remains an open problem. In particular, it involves the study of inverse spectral problems for the matrices $L$ appearing in the Lax representations for such systems, as well as the proof of results similar to Theorem 4. We plan to address these issues in our future work.

The author thanks Prof. Damianou for useful discussions and reference to [11].

\section{Appendix}

Proposition. The following identity holds for $k \geqslant 2$ and $a_{i, j} \in \mathbb{C}$ :

$$
\begin{gathered}
\left|\begin{array}{cccc}
a_{1,1} & a_{1,2} & \ldots & a_{1, k+1} \\
a_{2,1} & a_{2,2} & \ldots & a_{2, k+1} \\
\vdots & \vdots & \ldots & \vdots \\
a_{k+1,1} & a_{k+1,2} & \ldots & a_{k+1, k+1}
\end{array}\right|\left|\begin{array}{ccc}
a_{2,1} & \ldots & a_{2, k-1} \\
\vdots & \ldots & \vdots \\
a_{k, 1} & \ldots & a_{k, k-1}
\end{array}\right|+ \\
+\left|\begin{array}{cccc}
a_{1,1} & \ldots & a_{1, k-1} & a_{1, k+1} \\
a_{2,1} & \ldots & a_{2, k-1} & a_{2, k+1} \\
\vdots & \ldots & \vdots & \vdots \\
a_{k, 1} & \ldots & a_{k, k-1} & a_{k, k+1}
\end{array}\right|\left|\begin{array}{cccc}
a_{2,1} & a_{2,2} & \ldots & a_{2, k} \\
a_{3,1} & a_{3,2} & \ldots & a_{3, k} \\
\vdots & \vdots & \ldots & \vdots \\
a_{k+1,1} & a_{k+1,2} & \ldots & a_{k+1, k}
\end{array}\right|= \\
=\left|\begin{array}{cccc}
a_{1,1} & a_{1,2} & \ldots & a_{1, k} \\
a_{2,1} & a_{2,2} & \ldots & a_{2, k} \\
\vdots & \vdots & \ldots & \vdots \\
a_{k, 1} & a_{k+1,2} & \ldots & a_{k, k}
\end{array}\right|\left|\begin{array}{cccc}
a_{2,1} & \ldots & a_{2, k-1} & a_{2, k+1} \\
a_{3,1} & \ldots & a_{3, k-1} & a_{3, k+1} \\
\vdots & \ldots & \vdots & \\
a_{k+1,1} & \ldots & a_{k+1, k-1} & a_{k+1, k+1}
\end{array}\right| .
\end{gathered}
$$


Proof. Using the expansion of the first determinant to the last row, we rearrange (A.1) as

$$
\begin{aligned}
& (-1)^{k+2}\left(\left|\begin{array}{cccc}
a_{1,2} & a_{1,3} & \ldots & a_{1, k+1} \\
a_{2,2} & a_{2,3} & \ldots & a_{2, k+1} \\
\vdots & \vdots & \ldots & \vdots \\
a_{k, 2} & a_{k, 3} & \ldots & a_{k, k+1}
\end{array}\right|\left|\begin{array}{cccc}
a_{2,1} & \ldots & a_{2, k-1} & 0 \\
\vdots & \ldots & \vdots & \vdots \\
a_{k, 1} & \ldots & a_{k, k-1} & 0 \\
0 & \ldots & 0 & a_{k+1,1}
\end{array}\right|-\right. \\
& -\left|\begin{array}{cccc}
a_{1,1} & a_{1,3} & \ldots & a_{1, k+1} \\
a_{2,1} & a_{2,3} & \ldots & a_{2, k+1} \\
\vdots & \vdots & \ldots & \vdots \\
a_{k, 1} & a_{k, 3} & \ldots & a_{k, k+1}
\end{array}\right|\left|\begin{array}{cccc}
a_{2,1} & \ldots & a_{2, k-1} & 0 \\
\vdots & \ldots & \vdots & \vdots \\
a_{k, 1} & \ldots & a_{k, k-1} & 0 \\
0 & \ldots & 0 & a_{k+1,2}
\end{array}\right|+\ldots+ \\
& \left.(-1)^{k-1}\left|\begin{array}{ccccc}
a_{1,1} & \ldots & a_{1, k-2} & a_{1, k} & a_{1, k+1} \\
a_{2,1} & \ldots & a_{2, k-2} & a_{2, k} & a_{2, k+1} \\
\vdots & \ldots & \vdots & \vdots & \vdots \\
a_{k, 1} & \ldots & a_{k, k-2} & a_{k, k} & a_{k, k+1}
\end{array}\right|\left|\begin{array}{cccc}
a_{2,1} & \ldots & a_{2, k-1} & 0 \\
\vdots & \ldots & \vdots & \vdots \\
a_{k, 1} & \ldots & a_{k, k-1} & 0 \\
0 & \ldots & 0 & a_{k+1, k-1}
\end{array}\right|\right)= \\
& =\left|\begin{array}{cccc}
a_{1,1} & a_{1,2} & \ldots & a_{1, k} \\
a_{2,1} & a_{2,2} & \ldots & a_{2, k} \\
\vdots & \vdots & \ldots & \vdots \\
a_{k, 1} & a_{k+1,2} & \ldots & a_{k, k}
\end{array}\right|\left|\begin{array}{cccc}
a_{2,1} & \ldots & a_{2, k-1} & a_{2, k+1} \\
a_{3,1} & \ldots & a_{3, k-1} & a_{3, k+1} \\
\vdots & \ldots & \vdots & \vdots \\
a_{k+1,1} & \ldots & a_{k+1, k-1} & 0
\end{array}\right|- \\
& -\left|\begin{array}{cccc}
a_{1,1} & \ldots & a_{1, k-1} & a_{1, k+1} \\
a_{2,1} & \ldots & a_{2, k-1} & a_{2, k+1} \\
\vdots & \ldots & \vdots & \vdots \\
a_{k, 1} & \ldots & a_{k, k-1} & a_{k, k+1}
\end{array}\right|\left|\begin{array}{cccc}
a_{2,1} & \ldots & a_{2, k-1} & a_{2, k+1} \\
a_{3,1} & \ldots & a_{3, k-1} & a_{3, k+1} \\
\vdots & \ldots & \vdots & \vdots \\
a_{k+1,1} & \ldots & a_{k+1, k-1} & 0
\end{array}\right|
\end{aligned}
$$

Denote its left-hand and right-hand sides by I and II, respectively. For I and II we get the following representations:

$$
\mathrm{I}=\left|\begin{array}{ccccccccc}
a_{1,1} & a_{1,2} & \ldots & a_{1, k-1} & a_{1, k} & 0 & \ldots & 0 & a_{1, k+1} \\
a_{2,1} & a_{2,2} & \ldots & a_{2, k-1} & a_{2, k} & 0 & \ldots & 0 & a_{2, k+1} \\
\vdots & \vdots & \ldots & \vdots & \vdots & \vdots & \ldots & \vdots & \vdots \\
a_{k, 1} & a_{k, 2} & \ldots & a_{k, k-1} & a_{k, k} & 0 & \ldots & 0 & a_{k, k+1} \\
0 & 0 & \ldots & 0 & 0 & a_{2,1} & \ldots & a_{2, k-1} & 0 \\
\vdots & \vdots & \ldots & \vdots & \vdots & \vdots & \ldots & \vdots & \vdots \\
0 & 0 & \ldots & 0 & 0 & a_{k, 1} & \ldots & a_{k, k-1} & 0 \\
a_{k+1,1} & a_{k+1,2} & \ldots & a_{k+1, k-1} & 0 & 0 & \ldots & 0 & 0
\end{array}\right|
$$




$$
\mathrm{II}=\left|\begin{array}{ccccccccc}
a_{1,1} & a_{1,2} & \ldots & a_{1, k-1} & a_{1, k} & 0 & \ldots & 0 & a_{1, k+1} \\
a_{2,1} & a_{2,2} & \ldots & a_{2, k-1} & a_{2, k} & 0 & \ldots & 0 & a_{2, k+1} \\
\vdots & \vdots & \ldots & \vdots & \vdots & \vdots & \ldots & \vdots & \vdots \\
a_{k, 1} & a_{k, 2} & \ldots & a_{k, k-1} & a_{k, k} & 0 & \ldots & 0 & a_{k, k+1} \\
0 & 0 & \ldots & 0 & a_{2, k} & a_{2,1} & \ldots & a_{2, k-1} & a_{2, k+1} \\
\vdots & \vdots & \ldots & \vdots & \vdots & \vdots & \ldots & \vdots & \vdots \\
0 & 0 & \ldots & 0 & a_{k, k} & a_{k, 1} & \ldots & a_{k, k-1} & a_{k, k+1} \\
0 & 0 & \ldots & 0 & 0 & a_{k+1,1} & \ldots & a_{k+1, k-1} & 0
\end{array}\right| .
$$

Applying elementary transformations, we consequently find

$$
\mathrm{II}=\left|\begin{array}{ccccccccc}
a_{1,1} & a_{1,2} & \ldots & a_{1, k-1} & a_{1, k} & 0 & \ldots & 0 & a_{1, k+1} \\
a_{2,1} & a_{2,2} & \ldots & a_{2, k-1} & a_{2, k} & 0 & \ldots & 0 & a_{2, k+1} \\
\vdots & \vdots & \ldots & \vdots & \vdots & \vdots & \ldots & \vdots & \vdots \\
a_{k, 1} & a_{k, 2} & \ldots & a_{k, k-1} & a_{k, k} & 0 & \ldots & 0 & a_{k, k+1} \\
-a_{2,1} & -a_{2,2} & \ldots & -a_{2, k-1} & 0 & a_{2,1} & \ldots & a_{2, k-1} & 0 \\
\vdots & \vdots & \ldots & \vdots & \vdots & \vdots & \ldots & \vdots & \vdots \\
-a_{k, 1} & -a_{k, 2} & \ldots & -a_{k, k-1} & 0 & a_{k, 1} & \ldots & a_{k, k-1} & 0 \\
0 & 0 & \ldots & 0 & 0 & a_{k+1,1} & \ldots & a_{k+1, k-1} & 0
\end{array}\right|=\mathrm{I} .
$$

\section{References}

[1] Kac, M. and van Moerbeke, P., On an Explicitly Soluble System of Nonlinear Differential Equations Related to a Certain Toda Lattice, Adv. Math., 1975, vol. 16, no. 2, pp. 160-169.

[2] Moser, J., Three Integrable Hamiltonian Systems Connected with Isospectral Deformations, Adv. Math., 1975, vol. 16, no. 2, pp. 197-220.

[3] Akhiezer, N. I., The Classical Moment Problem and Some Related Questions in Analysis, Edinburgh: Oliver \& Boyd, 1965.

[4] Akhiezer, N. I. and Glazman, I. M., Theory of Linear Operators in Hilbert Space, New York: Dower, 1993.

[5] Babelon, O., Continuum Limit of the Volterra Model, Separation of Variables and Non-Standard Realizations of the Virasoro Poisson Bracket, Comm. Math. Phys., 2006, vol. 266, no. 3, pp. 819-862.

[6] Berezanskii, Ju. M., Expansions in Eigenfunctions of Selfadjoint Operators, Transl. Math. Monogr., vol. 17, Providence, R.I.: AMS, 1968.

[7] Berezanski, Yu. M., The Integration of Semi-Infinite Toda Chain by Means of Inverse Spectral Problem, Rep. Math. Phys., 1986, vol. 24, no. 1, pp. 21-47.

[8] Barrios Rolanía, D., Branquinho, A., and Foulquié Moreno, A., Dynamics and Interpretation of Some Integrable Systems via Multiple Orthogonal Polynomials, J. Math. Anal. Appl., 2010, vol. 361, no. 2, pp. 358-370.

[9] Bogoyavlensky, O. I., Breaking Solitons. Nonlinear Integrable Equations, Moscow: Nauka, 1991 (Russian).

[10] Damianou, P. A., The Volterra Model and Its Relation to the Toda Lattice, Phys. Lett. A, 1991, vol. 155 , nos. $2-3$, pp. $126-132$. 
[11] Damianou, P.A. and Loja Fernandes, R., From the Toda Lattice to the Volterra Lattice and Back, Rep. Math. Phys., 2002, vol.50, no.3, pp.361-378.

[12] Osipov, A., On Some Issues Related to the Moment Problem for the Band Matrices with Operator Elements, J. Math. Anal. Appl., 2002, vol. 275, no. 2, pp.657-675.

[13] Osipov, A.S., On a Determinant Criterion for the Weak Perfectness of Systems of Functions Holomorphic at Infinity and Their Connection with Some Classes of Higher-Order Difference Operators, Fundam. Prikl. Mat., 1995, vol. 1, no.3, pp. 711-727 (Russian).

[14] Osipov, A. S., Discrete Analog of the Korteweg-de Vries (KdV) Equation: Integration by the Method of the Inverse Problem, Math. Notes, 1994, vol. 56, no. 6, pp. 1312-1314; see also: Mat. Zametki, 1994, vol. 56, no. 6, pp. 141-144.

[15] Magri, F., A Simple Model of the Integrable Hamiltonian Equation, J. Math. Phys., 1978, vol. 19, no. 5, pp. 1156-1162.

[16] Morosi, C. and Pizzocchero, L., On the Continuous Limit of Integrable Lattices: 1. The KacMoerbeke System and KdV Theory, Comm. Math. Phys., 1996, vol. 180, no. 2, pp. 505-528.

[17] Yurko, V.A., On Higher-Order Difference Operators, J. Differ. Equations Appl., 1995, vol. 1, no. 4, pp. 347-352.

[18] Gantmacher, F. R., The Theory of Matrices: Vol. 2, New York: Chelsea, 2000.

[19] Gekhtman, M., Hamiltonian Structure of Non-Abelian Toda Lattice, Lett. Math. Phys., 1998, vol. 46, no. 3, pp. 189-205.

[20] Volkov, A. Yu., Hamiltonian Interpretation of the Volterra Model, J. Soviet Math., 1989, vol.46, no. 1, pp.1576-1581; see also: Zap. Nauchn. Sem. Leningrad. Otdel. Mat. Inst. Steklov. (LOMI), 1986, vol. 150, pp. 17-25, 218. 